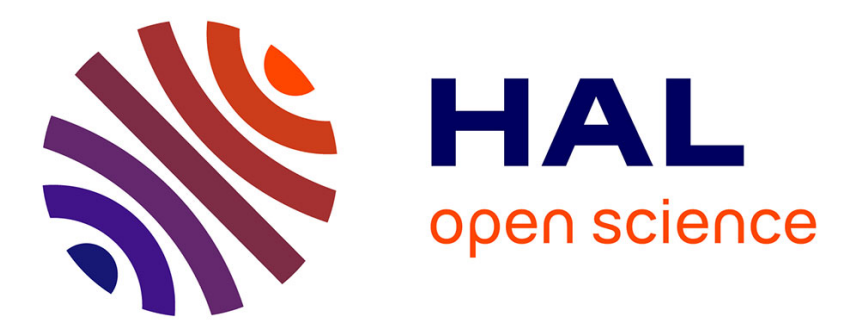

\title{
Restoration of hyperspectral astronomical data from Integral field spectrograph
}

Ferréol Soulez, Sébastien Bongard, Éric Thiébaut, Roland Bacon

\section{To cite this version:}

Ferréol Soulez, Sébastien Bongard, Éric Thiébaut, Roland Bacon. Restoration of hyperspectral astronomical data from Integral field spectrograph. WHISPERS'11, Jun 2011, Lisbonne, Portugal. pp.CDROM. hal-00618678

\section{HAL Id: hal-00618678 https://hal.science/hal-00618678}

Submitted on 2 Sep 2011

HAL is a multi-disciplinary open access archive for the deposit and dissemination of scientific research documents, whether they are published or not. The documents may come from teaching and research institutions in France or abroad, or from public or private research centers.
L'archive ouverte pluridisciplinaire HAL, est destinée au dépôt et à la diffusion de documents scientifiques de niveau recherche, publiés ou non, émanant des établissements d'enseignement et de recherche français ou étrangers, des laboratoires publics ou privés. 


\title{
RESTORATION OF HYPERSPECTRAL ASTRONOMICAL DATA FROM INTEGRAL FIELD SPECTROGRAPH
}

\author{
Ferréol Soulez $^{1,2}$, Sébastien Bongard ${ }^{3}$, Éric Thiébaut ${ }^{2}$, Roland Bacon ${ }^{2}$ \\ ${ }^{1}$ Centre Commun de Quantimetrie - \\ Université Lyon $1-8$ avenue Rockefeller, \\ 69373 Lyon cedex 08, France \\ ferreol.soulez@univ-lyon1.fr
${ }^{2}$ Centre de Recherche Astrophysique de ${ }^{3}$ Laboratoire de Physique Nucléaire et de Lyon - CNRS-UMR 5574 - Université Hautes Energies CNRS UMR 7585 - Lyon 1 - ENS Lyon - avenue Charles Université Paris 6 - Université Paris 7 - André, 69561 Saint-Genis Laval cedex, place Jussieu 75252 Paris Cedex 05, France.

France

\begin{abstract}
In this paper we present a method for hyper-spectral image restoration for integral field spectrographs (IFS) data. It takes advantage of all the spectral and spatial correlations in the observed scene to enhance the spatial resolution. We illustrate this method with simulations coming from the Multi Unit Spectroscopic Explorer (MUSE) instrument. It shows the clear increase of the spatial resolution provided by our method as well as its denoising capability.
\end{abstract}

Index Terms - Inverse problems, Deconvolution, Image restoration, ntegral field spectrograph,Astronomy.

\section{INTRODUCTION}

In the last decade, the integral field spectrographs (IFS) have become a popular tool for astronomical observation. Such instruments are now installed on all the main optical telescope facilities around the world. They provide spatially resolved spectra of a whole region of the sky, yielding $(\boldsymbol{\theta}, \lambda)$ data cubes - with $\theta$ the 2D angular position and $\lambda$ the wavelength that have several hundreds of wavelength bins. With IFS, astronomical data enters the hyper-spectral era. Dedicated image reconstruction techniques are thus needed to take full advantage of the data gathered by these instruments. Because the light is spread out on multiple channels instead of being integrated on a single image, the information content is increased at the cost of a lower signal to noise or achievable resolution for the same exposure time. Furthermore, atmospheric turbulence and instrumental response often spatially blur the observations, degrading the spatial resolution.

First attempts to restore multi-channel images consisted in applying classical 2D restoration techniques like Wiener filter or Richardson-Lucy algorithm on each individual channel. The caveat of these approaches is to ignore the natural spectral correlations present in the data. The first restoration technique specifically dedicated to multichannel data [?] was a MMSE restoration filter based on the assumption that signal autocorrelation is spatially and spectrally separable. This assumption was later relaxed[?] and many other multichannel linear restoration filters have been proposed since. More recently, Fourier/Wavelet restoration techniques have been adapted to multispectral data[?, ?]. Combining demixing and restoration some authors[?, ?] achieve enhanced spatial resolution given the strong assumption that the observed scene is composed of only a few materials with unknown spectrum.

Most of the work on restoration of multi-spectral images is dedicated to remote sensing and color (RGB) images. Those methods can't easily be directly applied to astronomical data with its specific features like large dynamic range and strong sharp features (for example narrow emission lines or peaked sources). To the best of our knowledge, restoration techniques for multi-spectral astronomical images have only been proposed for $(x, \lambda)$ data (slit spectrography) $[?, ?]$ or $(x, y, \lambda)$ data composed of slit spectrography scans [?]. At the current time, no similar techniques have been proposed for hyperspectral data observed simultaneously via an Integral Field Spectrograph (IFS).

We propose a new deconvolution method based on an inverse problem approach. It is very generic and exploits intrinsic continuities of hyper-spectral data. We suppose that a good estimation of the point spread function (PSF) is provided by other means (e.g. by calibration on the telescope guiding stars or on information from the adaptive optics system) and defer the blind deconvolution problem to a later time.

Our approach will be illustrated on data provided by the MUSE IFS simulator. Still in construction, the MUSE IFS[?] will be installed on the ESO Very Large Telescope (VLT). It is a "slicer" based IFS that covers in its wide field mode a $60^{\prime \prime} \times 60^{\prime \prime}$ spectroscopic field-of-view subdivided into a grid of about $300 \times 300$ spatial elements (spaxels). To each spaxel corresponds a spectrum, obtained by dispersing the light on 3463 equally spaced spectral bins from $480 \mathrm{~nm}$ to $930 \mathrm{~nm}$.

\section{PROBLEM FORMULATION}

We consider an observed data cube $\boldsymbol{y}$ of $N_{\lambda}$ monochromatic images of $N_{\Omega}$ pixels. We model it from the parameters $\boldsymbol{x}$ 
following the equation:

$$
\boldsymbol{y}=\mathbf{H} \cdot \boldsymbol{x}+\boldsymbol{e} .
$$

with $e$ the noise vector, and $\mathbf{H}$ the linear operator which approximates the convolution by the effective PSF and the sampling by the detector.

The PSF is assumed to be spatially shift invariant but its shape may vary with the wavelength. Similarly wavelengthwise PSF's may be centered at a location $\boldsymbol{\theta}_{\lambda}$ which depends on the wavelength so as to account for imperfect instrumental alignment and atmospheric differential refractive index (ADR). Furthermore PSF is not necessarily normalized in order to account for the variable throughput (atmospheric and instrumental transmission).

The parameters $\boldsymbol{x}$ describe the object 3-D distribution $I_{\text {obj }}(\boldsymbol{\theta}, \lambda)$ with a finite number of coefficients by means of expansion onto a basis of interpolation functions. The angular and spectral step sizes can be chosen to match the effective angular and spectral resolutions, in order to reduce the number of model parameters. We chose to control the effective number of free parameters by means of regularization and to take the same angular and spectral grid resolution than the data. The model parameters then simplify after discretization:

$$
x_{\boldsymbol{k}, \ell}=I_{\mathrm{obj}}\left(\boldsymbol{\theta}_{\boldsymbol{k}}, \lambda_{\ell}\right),
$$

where $\lambda_{\ell}$ is the effective wavelength in the $\ell$-th spectral channel and $\boldsymbol{\theta}_{\boldsymbol{k}}$ is the $k$-th angular position in an evenly spaced rectangular grid of pixels.

\section{MAXIMUM A POSTERIORI APPROACH}

Deconvolution is a typical ill-posed problem [?] which can be solved by adding priors in a classical Maximum A Posteriori (MAP) approach. This is achieved by estimating the object $\boldsymbol{x}^{+}$that minimizes the cost function $f(\boldsymbol{x})$ :

$$
\begin{aligned}
\boldsymbol{x}^{+} & =\underset{\boldsymbol{x}}{\arg \min } f(\boldsymbol{x}), \\
f(\boldsymbol{x}) & =f_{\text {data }}(\boldsymbol{x})+f_{\text {prior }}(\boldsymbol{x}) .
\end{aligned}
$$

This cost function $f(\boldsymbol{x})$ is the sum of a likelihood penalty $f_{\text {data }}(\boldsymbol{x})$ ensuring the agreement between the model and the data $\boldsymbol{y}$, and a regularization penalty $f_{\text {prior }}(\boldsymbol{x})$ introducing subjective a priori knowledge about the object.

\subsection{Likelihood and Noise Statistics}

Assuming Gaussian noise, the likelihood penalty reads:

$$
f_{\text {data }}(\boldsymbol{x})=[\boldsymbol{y}-\mathbf{H} \cdot \boldsymbol{x}]^{\mathrm{T}} \cdot \mathbf{W}_{\mathrm{err}} \cdot[\boldsymbol{y}-\mathbf{H} \cdot \boldsymbol{x}],
$$

where the weighting matrix $\mathbf{W}_{\text {err }}=\mathbf{C}_{\text {err }}^{-1}$ is the inverse of the angular-spectral covariance of the noise and approximations.
Assuming uncorrelated noise, $\mathbf{W}_{\text {err }}$ is diagonal and Eq. (5) simplifies to:

$$
f_{\text {data }}(\boldsymbol{x})=\sum_{j, \ell} w_{j, \ell}[\boldsymbol{y}-\mathbf{H} \cdot \boldsymbol{x}]_{j, \ell}^{2}
$$

where $1 / w_{j, \ell}$ is the noise variance of the measurements at pixel $j$ and channel $\ell$. This model can cope with nonstationary noise and can be used to express confidence on measurements on each pixel of the data. Since unmeasured data can be considered as having infinite variance, we can readily deal with missing or bad pixels as follows:

$$
w_{j, \ell} \stackrel{\text { def }}{=} \begin{cases}\operatorname{Var}\left(y_{j, \ell}\right)^{-1} & \text { if } y_{j, \ell} \text { is measured } \\ 0 & \text { otherwise }\end{cases}
$$

This treatment of missing data is consistent because (i) it consistently accounts for unmeasured data and bad pixels, and (ii) it allows to properly expand the synthesized f.o.v. to avoid field aliasing and border artifacts caused by convolution using Fourier transform.

Except for very low detector noise $\left(<\right.$ few $e^{-}$per pixel), we can approximate the total noise (Gaussian detector noise plus Poisson noise) by a non stationary uncorrelated Gaussian noise [?]:

$w_{j, \ell} \stackrel{\text { def }}{=} \begin{cases}\left(\gamma \max \left(y_{j, \ell}, 0\right)+\sigma_{j, \ell}^{2}\right)^{-1} & \text { if } y_{j, \ell} \text { is measured, } \\ 0 & \text { otherwise, }\end{cases}$

where $\gamma$ accounts for the quantization factor of the detector and $\sigma_{j, \ell}^{2}$ is the variance of other approximately Gaussian noise on the pixel $(j, \ell)$, like for example read-out noise.

\subsection{Regularization}

Astronomical data is mainly composed of bright objects (stars, galaxy) over a flat background. Most of the quite large MUSE field of view will thus contain only background. As a consequence, the data will be intrinsically spatially sparse. This spatial sparsity prior can be enforced by means of mixed norms[?, ?]:

$$
f_{\text {sparsity }}(\boldsymbol{x})=\sum_{\boldsymbol{k}}\left[\sqrt{\sum_{\ell} \boldsymbol{x}_{\boldsymbol{k}, \ell}^{2}+\epsilon^{2}}-\epsilon\right]
$$

where $\epsilon$ is a small real number $\left(\epsilon \approx 10^{-9}\right)$ that ensures the derivability in 0 (hyperbolic approximation of the $\ell_{1}$ norm). This regularization enforces spatial sparsity and spectral correlation since it favors when bright spaxels in each spectral channel are at the same spatial location.

The regularization defined in Eq. (8) does not ensure the spectral continuity of the solution whereas in practice this solution should be relatively smooth along the spectral dimension excepted near emission and absorption lines. For that 
reason we introduce an additional regularization function:

$$
f_{\text {spectral }}(\boldsymbol{x})=\sum_{\boldsymbol{k}, \ell}\left[\sqrt{\left(\boldsymbol{x}_{\boldsymbol{k}, \ell}-\boldsymbol{x}_{\boldsymbol{k}, \ell-1}\right)^{2}+\zeta^{2}}-\zeta\right]
$$

This regularization tends to smooth the spectra $x_{k}$ but preserve discontinuity where $\left|\boldsymbol{x}_{\boldsymbol{k}, \ell}-\boldsymbol{x}_{\boldsymbol{k}, \ell-1}\right| \gg \zeta$. This situation is for example encountered at absorption or emission lines, which shall not be smoothed.

Owing to the large difference of dynamical range between spectral channel of astronomical images, these regularizations lead to over-regularization of bright features or under-regularization of fainter ones. For that reason, we rather suggest to apply these regularization functions to an spectrally whitened object $\boldsymbol{x}^{\prime}$ :

$$
x_{k, \ell}^{\prime}=x_{k, \ell} / s_{\ell}
$$

with $s_{\ell}=\left\langle x_{k, \ell}\right\rangle_{k}$ the spatially averaged object spectrum \langle\rangle$_{k}$ denotes averaging over pixel index $k$. To avoid dealing with non-linear regularization, we estimate the mean object spectrum directly from the data:

$$
s_{\ell}=\left\langle y_{j, \ell}\right\rangle_{j} / \eta_{\ell}
$$

with $\eta_{\ell}=\eta\left(\lambda_{\ell}\right)$ the effective throughput in $\ell$-th spectral channel. Note that this approximation is justified because we do not attempt to perform spectral deconvolution and because we use the same wavelengths grid for the sought distribution and the data.

Finally our regularization penalty writes:

$$
f_{\text {prior }}(\boldsymbol{x})=\alpha f_{\text {sparsity }}\left(\boldsymbol{x}^{\prime}\right)+\beta f_{\text {spectral }}\left(\boldsymbol{x}^{\prime}\right) .
$$

where $\alpha$ and $\beta$ are the hyper-parameters that have to be tuned to set the importance of the priors.

\section{ALGORITHM DESCRIPTION}

In the presented experiments, we suppose that there is no cross-talk between spectral channels, the matrix $\mathbf{H}$ is block diagonal, and since the PSF is isoplanatic, applying $\mathbf{H}$ consists in $N_{\lambda}$ discrete spatial convolutions, one for each spectral channel. Due to the convolution process, flux from the object just outside of the field of view is measured inside data. To take this fact correctly into account, the estimated object has to be spatially larger than the observed field of view. At least half of the PSF support must be added on each side of the observed field of view to form the restored field of view. Furthermore, as in practice the convolution is computed using FFT, the same field extrapolation prevents border artifacts due to the circulant approximation. In the end, the application of $\mathbf{H}$ requires $N_{\lambda}$ spatial FFTs.

The hyper-parameters $\alpha$ and $\beta$ are estimated by trial and error. The deconvolved data cube $\boldsymbol{x}^{+}$is the solution of Eq. (3). It requires the minimization of the cost function $f(\boldsymbol{x})$ that involves a large number of parameters $\left(>1 \times 10^{6}\right)$. To that end, we use the VMLM-B algorithm[?] which is a limited memory variant of the variable metric method with BFGS updates[?]. This algorithm has proved its effectiveness for image reconstruction and only requires the computation of the penalty function being minimized together with its gradient. The memory requirement is a few times the size of the problem.

\section{RESULTS}

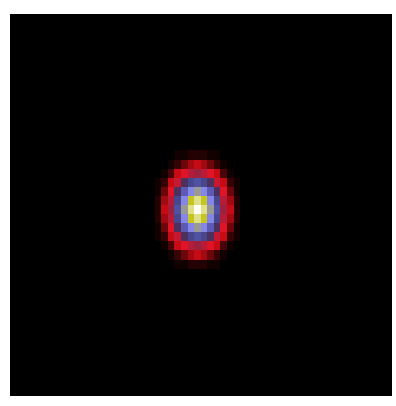

(a) PSF at $\lambda=480 \mathrm{~nm}$

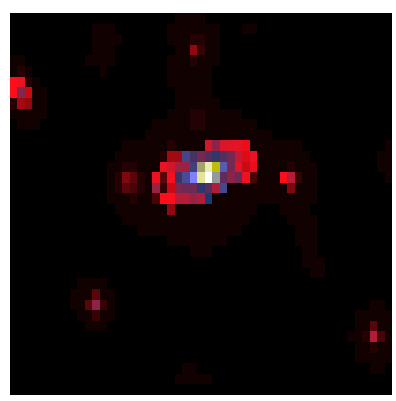

(c) Reconstruction

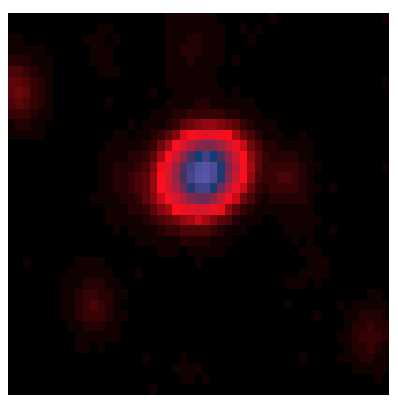

(b) Raw image

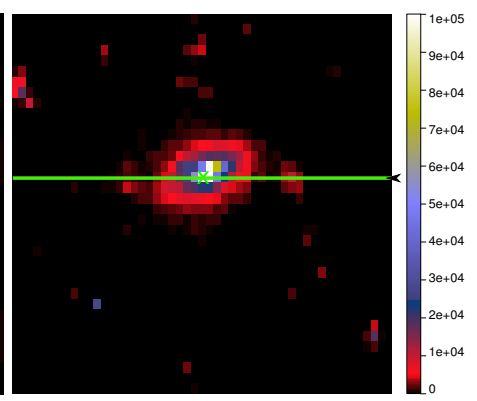

(d) True image
Fig. 1. Images from the data, the deconvolution and the ground truth summed along the spectral dimension.

The quality of the presented algorithm was assessed on data from the MUSE IFS simulator. This data is a part of $51 \times 36$ spaxels (pixels size: $0^{\prime \prime} .2 \times 0^{\prime \prime} .2$ ) of the whole MUSE data cube. It contains 3463 spectral channels comprised between $480 \mathrm{~nm}$ and $930 \mathrm{~nm}$. The PSF, shown on Fig. 1(a) is a Gaussian with a full width at half maximum that varies from 0 . 75 (3.75 pixels) at the red end to 0 ".92 (4.6 pixels) at the blue end. In Fig. 1(b), the data summed all over the channels is displayed so that it can be compared with the ground truth in Fig. 1(d). The corresponding deconvolution presented in Fig. 1(c), clearly illustrates the gain of resolution provided by our method. Both the shapes of the central galaxy and of the one near the upper left corner are recovered. Figure ?? displays the spectra of the central spaxel of the galaxy from the data (blue), the deconvolution (dashed red) and the ground truth (black). Even though regularizations introduce some ex- 
pected bias, the restored spectra are close to the ground truth: most of the spectral features are preserved. We display in Figures ??(b-c) spectral cuts through the heart of the central galaxy materialized by the yellow line in Fig.1(d). These figures show $(\theta, \lambda)$ images zoomed between $568 \mathrm{~nm}$ and $576 \mathrm{~nm}$ for the data, the restoration and the ground truth. These plots show the resolution gain provided by our algorithm: both brighter structures are separated, with the spectrum at $\theta=43$ th column visible in the restoration that was not visible in the data. Futhermore, the noise observed around the galaxy has been drasticaly reduced by our method.

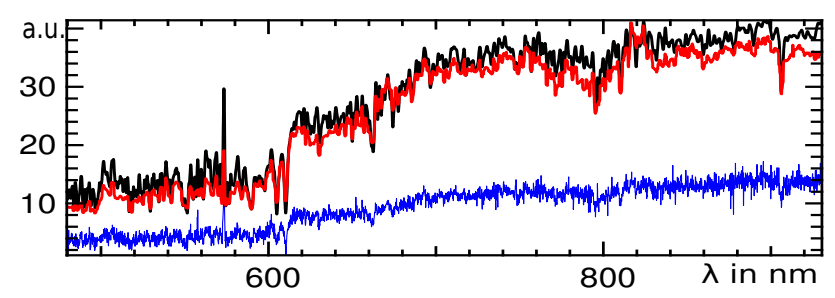

(a) Spectra of the spaxel materialized by the green 'x' in Fig. 1(d) for the data (blue), the deconvolution (red) and the ground truth (black).

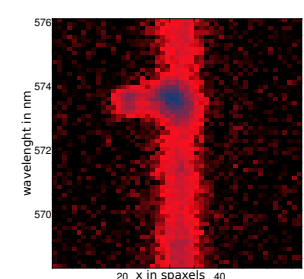

(b) Raw image.

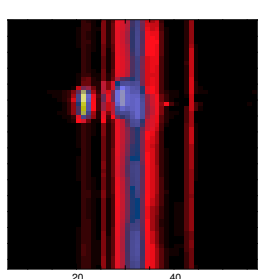

(c) Reconstruction

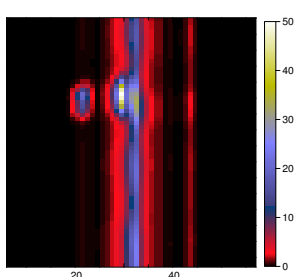

(d) True image
Fig. 2. $(\theta, \lambda)$ images of the cut materialized by the yellow line in Fig. 1(d) magnified between $568 \mathrm{~nm}$ and $576 \mathrm{~nm}$.

\section{CONCLUSION}

We present a new method for deconvolving hyperspectral data. By exploiting both spatial and spectral correlations present in the data, our method provides a strong spatial resolution enhancement and an effective denoising along the spectral dimension (given that we suppose the absence of channel cross-talk). Its deblurring performance is assessed on simulations. The results clearly demonstrate the advantages to process the whole data-cube instead of processing each wavelength channel independently. This increase of the spatial resolution would be of special interests for many astronomical subject such as the study of the kinematics of galaxies or weak lensing surveys, for which the true shape of the galaxy uncontaminated by the observational blur is of special interest.

During this work, Ferréol Soulez has been funded by the French Agence Nationale de la Recherche on the MiTiV project (ANR-09-EMER-008-01).

\section{REFERENCES}

[1] B. R. Hunt and O. Kubler, "Karhunen-Loeve multispectral image restoration. i: Theory," IEEE Trans. Acoust., Speech, Signal Process., vol. 32, no. 3, pp. 592-600, 1984.

[2] N.P. Galatsanos and R.T. Chin, "Digital restoration of multichannel images," IEEE Trans. Acoust., Speech, Signal Process., vol. 37, no. 3, pp. 415-421, 1989.

[3] A Benazza-Benyahia and J-C. Pesquet, "Multichannel image deconvolution in the wavelet transform domain," in $E U$ SIPCO, 2006.

[4] A. Duijster et al., "Wavelet-Based EM algorithm for Multispectral-Image restoration," IEEE Trans. Geosci. Remote Sens., vol. 47, no. 11, pp. 3892-3898, 2009.

[5] T. Akgun et al., "Super-resolution reconstruction of hyperspectral images," IEEE Trans. Image Process., vol. 14, no. 11, pp. 1860-1875, 2005.

[6] J. Bobin et al., "Sparsity constraints for hyperspectral data analysis: linear mixture model and beyond," in SPIE Conference Series, 2009, vol. 7446, p. 42.

[7] F. Courbin et al., "A method for spatial deconvolution of spectra," The Astrophysical Journal, vol. 529, pp. 1136, 2000.

[8] L. B. Lucy and J. R. Walsh, "Iterative techniques for the decomposition of long-slit spectra," The Astronomical Journal, vol. 125, pp. 2266, 2003.

[9] T. Rodet et al., "Data inversion for Over-Resolved spectral imaging in astronomy," IEEE J. Sel. Topics Signal Process., vol. 2, no. 5, pp. 802-811, 2008.

[10] F. Henault et al., G. Gilmore, O. Le Fevre, J.P. Lemonnier, S. Lilly, et al., "MUSE, a second-generation integral-field spectrograph for the VLT," in Proceedings of SPIE, 2003, vol. 4841, pp. 1096-1107.

[11] M. Bertero and P. Boccacci, Introduction to Inverse Problems in Imaging, Taylor \& Francis, 1998.

[12] L. Mugnier et al., "Mistral: a myopic edge-preserving image restoration method, with application to astronomical adaptiveoptics-corrected long-exposure images," J. Opt. Soc. Am. A, vol. 21, no. 10, pp. 1841-1854, 2004.

[13] M. Fornasier and H. Rauhut, "Recovery Algorithms for Vector-Valued Data with Joint Sparsity Constraints," SIAM Journal on Numerical Analysis, vol. 46, pp. 577, 2008.

[14] M. Kowalski and B. Torrésani, "Sparsity and persistence: mixed norms provide simple signal models with dependent coefficients," Signal, Image and Video Processing, vol. 3, pp. 251-264, 092009.

[15] E. Thiébaut, "Optimization issues in blind deconvolution algorithms," in Astronomical Data Analysis II., Jean-Luc Starck, Ed., dec 2002, vol. 4847, pp. 174-183.

[16] J. Nocedal and S.J. Wright, Numerical optimization, Springer, 1999. 